Brit. J. vener. Dis. (1956), 32, 40.

\title{
SEROLOGICAL, NEPHELOMETRICAL, AND STATISTICAL STUDIES ON THE EMPLOYMENT OF SYNTHETIC LECITHIN IN CARDIOLIPIN ANTIGENS*
}

\author{
BY \\ ALICE REYN, MICHAEL WEIS BENTZON, AND JULIE HARTMANN \\ From the Serodiagnostic and Statistical Departments, Statens Seruminstitut, Copenhagen \\ Director : J. Ørskov
}

Cardiolipin antigens, used in the sero-diagnosis of syphilis, are mixtures of the phospholipids, cardiolipin, and purified natural lecithin (beefheart or egg), and usually also contain cholesterol.

In 1949 Baer and Kates $(1949,1950)$ succeeded in the synthesis of $\mathrm{L}-\alpha$-dimyristoyl lecithin and in the following years other types of synthetic lecithin were produced, including a D- $\alpha$-dimyristoyl lecithin (Baer, 1952 ; Baer and Martin, 1951 ; Baer and Maurukas, 1952). The new substances were serologically evaluated by Rosenberg (1949), Kline (1950), Faure and Maréchal (1952), and Tonks and Allen (1953), who all reported that the synthetic products were usable in the preparation of cardiolipin antigens, that is, for the VDRL microflocculation test, the Hinton, Rein-Bossak, and Kline microflocculation tests, and the Kolmer and Débain complement-fixation tests. Yet the antigens containing synthetic lecithin were generally weaker than antigens prepared with natural lecithin (Faure and Maréchal, 1952). L- and D-lecithins were found equally active (Tonks and Allen, 1953).

In a preliminary report Rosenberg (1949) found synthetic $L-\alpha$-dipalmitoyl lecithin serologically active, and Kline (1950) reported that L- $\alpha$-dimyristoyl " acted well " as an antigen component with cardiolipin in the sero-diagnosis of syphilis. Mixtures of nine to twelve parts synthetic lecithin to one part cardiolipin gave results similar in sensitivity and specificity to those of optimal cardiolipin antigens with natural lecithins.

Faure and Maréchal (1952) found the antigens less sensitive when the synthetic lecithin was used in the same proportions as the natural lecithins, but they reported that the sensitivity could be increased by decreasing the lecithin content. However, neither the ethanolic antigens nor the saline suspensions prepared from these antigens kept very well. In addition, nonspecific results were obtained with a 1-week-old Kline antigen.

* Received for publication October 24, 1955.
In VDRL slide test experiments Tonks and Allen (1953), employing the same percentages (weight/volume) in the test and reference antigens, found that the sensitivity of the test antigens containing synthetic L- $\alpha$ dimyristoyl lecithin increased in the first 4 hours. However, if heated for $5 \mathrm{~min}$. at $56^{\circ} \mathrm{C}$., stable suspensions of maximum sensitivity were readily obtained, but the most reactive preparations were not quite as sensitive as the VDRL reference antigen. In the Kolmer test levels of sensitivity close to that of Kolmer lipoidal antigen were obtained when the following composition was used : cardiolipin 0.0175 per cent.; synthetic lecithin 0.2 per cent. ; cholesterol 0.3 per cent.

In 1953 Baer produced a purified, crystalline L- $\alpha$ dimyristoyl lecithin. Portions of this product were required by WHO, Geneva, for serological evaluation at different laboratories, including the WHO Reference Centre, Statens Seruminstitut, Copenhagen. The results of part of the experiments performed in Copenhagen were reported elsewhere (Reyn and Bentzon, 1956.) In complement-fixation and VDRL slide test experiments using equimolecular amounts of synthetic and natural (egg) lecithin, the antigens containing synthetic lecithin were less sensitive than those containing natural lecithin.

Kline (1954 a, b, 1955) has also tested the purified product and found it " to act very well" ; he concludes that the cardiolipin (C)/synthetic lecithin (SL) ratio, $10 / 9$, is optimal for use in his test. Four different lots of synthetic crystalline $\mathbf{L}$ - $\alpha$-dimyristoyl lecithin were very similar with respect to this optimal C/SL ratio ; this was in contrast to the variable optimal ratio of cardiolipin and natural lecithin.

Tonks, Allen and Fowler (1955), in a preliminary report, suggest that L- $\alpha$-dioleyl lecithin (unsaturated) may be even more useful than saturated $L-\alpha$-dimyristoyl lecithin.

The significance of the nature of the fatty acids contained in cardiolipin and lecithin was studied by Faure (1949), who investigated the serological and chemical effects of complete hydrogenization of cardiolipin and beef-heart lecithin. After hydrogenization both substances grew less soluble in ethanol. In serological tests (Kolmer's complement-fixation and Brown's microflocculation test (Brown, 1946) the 
specificity was found unaltered after hydrogenization, whereas the sensitivity was changed. The saturated lecithin resulted in decreased sensitivity when combined with either normal or saturated cardiolipin. Saturated cardiolipin gave slightly increased sensitivity when combined with untreated beef-heart lecithin. The changes in sensitivity were ascribed to changes in the affinity of the two substances for water on the one hand and cholesterol on the other, and perhaps also to changes in affinity for each other. In their report on synthetic lecithin, Faure and Maréchal (1952) ascribed the decreased sensitivity of antigens prepared with synthetic lecithin to the fact that this lecithin was saturated.

Tonks, Allen, and Fowler (1955) briefly report on serological experiments performed with "Pangborn lecithins" (which contain both saturated and unsaturated fatty acids), unsaturated synthetic ( $L-\alpha$-dioleyl), saturated synthetic ( $L-\alpha$-dimyristoyl), and unsaturated natural (L- $\alpha$-dipalmitoyl) lecithin, the latter being prepared from yeast (Tonks and Allen, 1955). In the VDRL slide flocculation test, the Pangborn lecithin (Pangborn, Maltaner, Tompkins, Beecher, Thompson, and Flynn, 1951) was more effective per unit weight than the other lecithins, and a smaller amount of the synthetic saturated lecithin than of either of the unsaturated lecithins was required.

Summarizing the above, we find that most authors agree that for optimal sensitivity the ratio between cardiolipin and lecithin (molecule by molecule) is apparently not the same for natural lecithins (egg or beef-heart) as for synthetic lecithins, whether saturated or unsaturated, purified or crude. The significance of varying the absolute concentrations of cardiolipin, synthetic lecithin, and cholesterol has not yet been submitted to a closer analysis. Generally, decreased sensitivity was observed with the use of equimolecular amounts of synthetic and natural lecithin. The unpurified lots of $D-\alpha-$ dimyristoyl lecithin showed bad keeping qualities (Faure and Maréchal, 1952), but the purified, crystalline lots give promise of improved keeping qualities (Faure, 1956 ; Reyn and Bentzon 1956).

\section{Present InVestigation}

In the present experimental series the effect of varying the $C / L$ ratio with constant cardiolipin and cholesterol concentration (involving a variation in absolute lecithin concentration) was investigated in quantitative complement-fixation experiments. In addition, nephelometric measurements were made of the light-scattering effects of the various antigen suspensions, including registration of maturation curves (Hartmann, Reyn, and Schmidt, 1953 ; Hartmann, 1955; Hartmann and Reyn, 1955).

Furthermore, the keeping qualities of the antigens containing various concentrations of synthetic lecithin were compared in complement-fixation and VDRL slide flocculation experiments with those of antigens containing natural egg lecithin. The effects of storage at room temperature for about 6 months and of storage at $56^{\circ} \mathrm{C}$. for about 4 months were investigated. The serological and nephelometric results were subjected to a statistical analysis.

\section{Material}

In December, 1953, 400 mg. synthetic, crystalline L- $\alpha$-dimyristoyl lecithin was received from Professor Baer, Toronto, Canada. The lecithin had been requested through WHO, Geneva, for serological testing at the WHO Reference Centre, Statens Seruminstitut, Copenhagen. The substance was kept in a dry condition at about $+4^{\circ}$ C. until February 8,1954 , when a $3 \mathrm{w} / \mathrm{v}^{*}$ per cent. solution in absolute ethanol was prepared. The lecithin was readily soluble in ethanol; and the resulting solution was clear and colourless.

Antigens.-On February 9, 1954, seven antigens were prepared : five complement-fixation (CF) antigens and two VDRL slide flocculation antigens. The International Reference Preparations for 1953 (IRP 1953) were used as references. The antigens were stored at room temperature when not otherwise indicated. The composition of the various antigens is given in the Table.

TABLE

COMPOSITION OF ANTIGENS

\begin{tabular}{|c|c|c|c|c|}
\hline \multirow{2}{*}{$\begin{array}{c}\text { Antigen } \\
\text { Numbers }\end{array}$} & \multicolumn{2}{|c|}{$\begin{array}{c}\text { Lecithin } \\
\text { (w/v per cent.) }\end{array}$} & \multirow{2}{*}{$\begin{array}{c}\text { Cardio- } \\
\text { lipin (IRP } \\
\text { 1953) } \\
\text { (w/v per } \\
\text { cent.) }\end{array}$} & \multirow{2}{*}{$\begin{array}{c}\text { Cholesterol } \\
\text { Pfanstiehl } \\
\text { precipitated } \\
\text { from } \\
\text { Ethanol } \\
\text { (w } / \mathbf{v} \text { per } \\
\text { cent.) }\end{array}$} \\
\hline & Synthetic & $\underset{1953)}{\text { Egg (IRP }}$ & & \\
\hline $\begin{array}{ll}\text { CF } & \text { Test } 1 \\
\text { CF } & \text { Test } 2 \\
\text { CF Test } 3 \\
\text { CF Test } 4 \\
\text { VDRL }\end{array}$ & $\begin{array}{l}0.039 \\
0.078^{*} \\
0.0875 \dagger \\
0.156 \\
0.255\end{array}$ & $\begin{array}{l}E \\
z\end{array}$ & $\begin{array}{l}0.0175 \\
0.0175 \\
0.0175 \\
0.0175 \\
0.03\end{array}$ & $\begin{array}{l}0.3 \\
0.3 \\
0 \cdot 3 \\
0 \cdot 3 \\
0.9\end{array}$ \\
\hline $\begin{array}{l}\text { CF } \\
\text { VDRL Reference }\end{array}$ & - & $\begin{array}{l}0.0875 \\
0.27\end{array}$ & $\begin{array}{l}0.0175 \\
0.03\end{array}$ & $\begin{array}{l}0.3 \\
0.9\end{array}$ \\
\hline
\end{tabular}

* Normal ; † Super-normal.

The molecular weight of the synthetic lecithin, i.e., dimyristoyl-L- $\alpha$-glyceryl-phosphoryl-choline (Baer and Kates, 1950), was calculated at 695.6 and the molecular weight of egg lecithin was calculated at $785, P=3.95$ per cent. (Pangborn and others, 1951). Antigens with compositions other than indicated in the Table were prepared by mixing test antigen No. 1 with varying amounts of ethanolic cardiolipin cholesterol solutions containing " normal " concentrations of these substances and no lecithin (see p. 42).

Sera.-Freeze-dried, positive syphilitic sera and fresh routine sera, positive and negative, were employed, the latter being negative in the Kahn Standard test. The five positive sera were derived from one case of secondary untreated syphilis, one case of newly treated latent syphilis, two treated cases of tertiary syphilis, and one case of old treated syphilis.

* $\mathbf{w} / \mathrm{v}=$ weight $/$ volume (weight : lecithin, cardiolipin, or cholestero volume : absolute ethanol.) 


\section{Method}

Quantitative Complement-fixation as Modified by Morch, 1933.-The method was recently described by Schmidt (1951) for use in experiments with cardiolipin antigen. Serum dilution steps were used as in the New York State CF method (Pangborn and others, 1951), i.e., parallel dilutions with a difference of 0.125 in logarithmic value between the tubes. The results were given as $\log _{10}$ titre values at 50 per cent. haemolysis, determined by the Kärber method (Finney, 1947). Preliminary complement $\left(C^{\prime}\right)$ titration experiments indicated that the same $C^{\prime}$ titre could be used in experiments with antigens containing synthetic lecithin as well as in experiments with the reference antigen. With the exception of the maturation experiments, the saline antigen suspensions were always allowed to stand or " mature" for 2 hours at room temperature before use (Lundbäck, 1952 ; Schmidt, Lundbäck, and Reyn, 1953 ; Schmidt and Lundbäck, 1954). After that time, viewed with the naked eye, the saline suspensions of the antigen containing " normal" concentration of synthetic lecithin appeared to have an opalescence similar to that of the reference antigen. All results were read by one technician and recorded by another.

VDRL Microflocculation.-Manual of Serological Tests for Syphilis, 1949. Federal Security Agency, Public Health Service Division of Venereal Diseases, Washington. The readings were performed as in the $\mathrm{CF}$ experiments and the results were given as $\log _{10}$ titre values corresponding to "dils".

In all experiments only one portion of each antigen was employed for all procedures ; all the experiments were performed within 4 hours of the inactivation of sera.

Nephelometric Technique.-The registration of the light scattered by antigen saline suspensions left standing for various periods at room temperature was performed by means of a Coleman Universal Spectrophotometer, Model 14, as previously described (Hartmann, 1955; Hartmann and Reyn, 1955 ; Hartmann and others, 1953).

\section{Results}

(a) CF Experiments.-In April, 1954, five freezedried syphilitic sera were repeatedly tested in $\mathrm{CF}$ experiments on 4 experimental days; in October, 1954, the same five sera were tested on 3 experimental days. The sera were titrated against the reference antigen and the three test antigens, 1, 2, and 4, containing half, " normal", and double concentrations of the synthetic lecithin (Table). Average titres for the repeated testing on different days were computed for each serum, as in the testings in April and in October, one set of titres for each series of experiments. The results are shown in Fig. 1 (opposite). The differences between the average titres found with the test antigens and those found with the reference antigen are plotted on the ordinate, the abscissa designating the lecithin concentration of the three test antigens. Each point on the Figure corresponds to an average difference and each serum has its own designation. It is evident that for each serum the difference between the reference and test antigens is correlated with the variation in lecithin concentration; this applies to the experiments performed in April as well as in October. It is also clear that the different sera register this variation in a different manner, some sera remaining comparatively unaffected by a halving of the lecithin concentration, others demonstrating this change very markedly. Thus, the curve for serum 109 is rather flat and that for serum 120 rather steep.

The test antigen with " normal" concentration of synthetic lecithin (No. 2) is somewhat less sensitive than the reference antigen, the total average titre for all five sera being 0.1 in $\log _{10}$ value less than that of the reference antigen. However, the test antigen with a double lecithin concentration (No. 4) is far less sensitive, showing a total average difference of about 0.35 from the reference antigen. The test antigen containing only " half normal" concentration (No. 1) shows a total average titre very close to that of the reference antigen.

The range of the deviation from the reference antigen for the individual sera is greatest for the test antigen with a double concentration, smaller for the test antigen with a half concentration, and smallest for that with " normal" concentration. For the antigens with a double concentration the range of deviation from the reference antigen is greater in October than in April.

Preliminary experiments with a series of lecithin concentrations between " normal " and half showed that the sensitivity increased gradually from " normal " to half concentration.

In order to investigate whether a further decrease in lecithin concentration would result in an antigen of the same average sensitivity and with the same range of deviation between the individual sera, antigens were prepared with $1 / 4,1 / 8$, and $1 / 16$ of the " normal" concentration of lecithin. In July, 1955, the same five freeze-dried sera that were tested in April and in October, 1954, were tested against these antigens and re-tested against the antigen with a half concentration of lecithin. The experiments were repeated on 3 consecutive days.

In Fig. 2 (opposite) the average differences between the titres obtained with the test antigens and those obtained with the reference antigen are plotted on the ordinate, the abscissa designating the lecithin concentration of the test antigens. The figures corresponding to double and normal concentration are averages from the April and October experiments; the figures corresponding to half concentration are averages from the April and October 

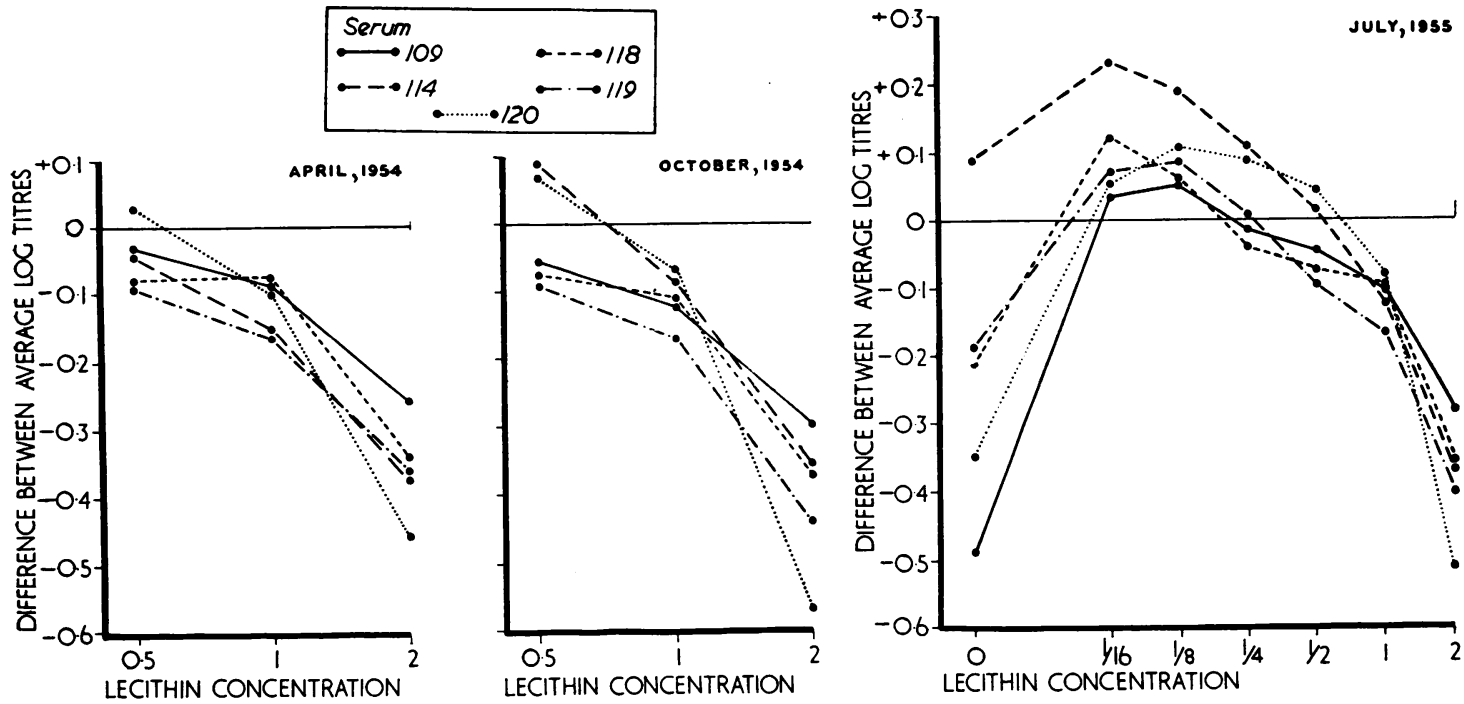

FIG. 1.-Comparison between the reference antigen and three test antigens with varied concentration of synthetic lecithin (normal concentration $=1 \cdot 0$ ). Experiments performed in April and October, 1954.

1954, and July, 1955, experiments, whereas the remaining figures are based on the most recent experiments only. Each point on the Figure corresponds to an average titre, and each serum has its own designation.

Lecithin concentration zero stands for an incomplete antigen (" Cardchol "), containing no lecithin, but cardiolipin and cholesterol in the same concentrations as used in the reference antigens. The results were obtained in previous experiments by Schmidt (1955a, b).

The average sensitivity increases with decreasing lecithin concentration down to about $1 / 8$ or $1 / 16$ of the "normal" concentration. The antigen without lecithin (Cardchol)' shows a lower average than the antigen containing $1 / 16$ of the "normal" concentration.

It is clear that the range of deviation from the reference antigen for the individual sera is the narrowest for the antigen with "normal " lecithin concentration, and that it increases on both sides of this concentration. The increase is only slight from " normal" to $1 / 8$ " normal" concentration, i.e., from 0.085 to $0.138 \log _{10}$ value, whereas the range for the Cardchol antigen is very wide $(0.578)$ and wider than that for the antigen with a double concentration $(0 \cdot 233)$.

In addition to the five syphilitic sera, two presumably biologic false positive (BFP) sera were tested; both sera were TPI* negative and were

\footnotetext{
* Treponema pallidum immobilization test (Nelson and Mayer, 1949).
}

FIG, 2-Comparison between the reference antigen and seven test antigens with varied concentration of synthetic lecithin (normal concentration $=1.0$ ). Experiments performed in April and October, 1954, and in July, 1955.

derived from acute respiratory cases. Some of the antigens showed zone phenomena with these sera, rendering it impossible to do anything but a rough estimation of the titres. These "titres" showed far greater variations from day to the day than the titres found in specific sera. The two BFP sera were not tested with antigens containing "normal" concentration of synthetic lecithin. Half lecithin concentration resulted in almost complete lack of reaction, but the reactivity greatly increased with decreasing lecithin concentration.

(b) Nephelometric Registration of the CF Antigen Suspensions.- Nephelometric maturation curves (Hartmann, 1955 ; Hartmann and Reyn, 1955; Hartmann and others, 1953) were registered for Cardchol antigen, reference antigen, and four test antigens (the three antigens with double, " normal ", and half lecithin concentration, and one test antigen with the same lecithin concentration as the reference antigen by absolute weight, i.e., about 10 per cent. more lecithin than "normally", as determined by molecular weight) on the curve designated " supernormal ".

In Fig. 3 (overleaf) the nephelometric values for the six antigen suspensions are plotted against time, the observation period being $30 \mathrm{~min}$. for all suspensions. The curve for double concentration is nearly horizontal with a slight tendency to a lower value after $5 \mathrm{~min}$. The other curves, including that of the reference antigen, rise during the experimental 


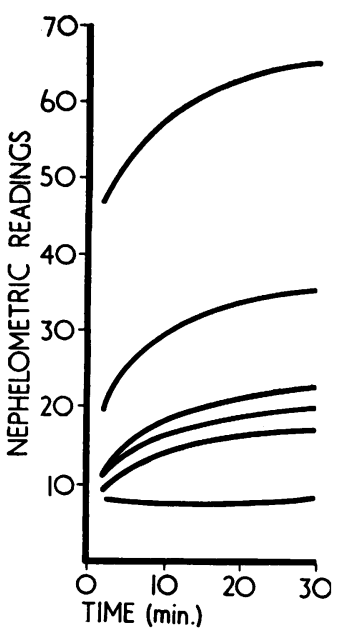

FIG. 3.-Nephelometric maturation curves for the reference antigen and five antigens with varied synthetic lecithin concentration.

period, having a tendency to flatten as time goes on. Thus, during the experimental period nephelometric maturation was not demonstrated with the antigen containing double concentration.

However, serological maturation (Lundbäck, 1952 ; Schmidt and others, 1953 ; Schmidt and Lundbäck, 1954), as measured by the average titre increase of three syphilitic sera tested against suspensions stored for different periods $\left(0, \frac{1}{2}, 1\right.$, 2,4 , and $18 \mathrm{hrs}$ ), was at least just as significant with this antigen as with the other antigens.

Considering the curves for the antigens containing synthetic lecithin and that for the Cardchol antigen, increasing nephelometric values are observed with decreasing lecithin concentration. In the abovementioned serological experiments, increasing sensitivity was observed with decreasing lecithin concentration down to about $1 / 8$ or $1 / 16$ of the " normal" concentration, whereas lecithin concentrations lower than this resulted in decreasing sensitivity. Thus, the nephelometric values and the sensitivity are only "positively correlated" for concentrations of synthetic lecithin higher than $1 / 8$ of the "normal". The curve for reference antigen is located slightly above that for the antigen with " normal" concentration of synthetic lecithin, and the reference antigen is also slightly more sensitive than this antigen.

(c) Keeping Qualities.-Aliquots of the reference and test antigens were stored at $56^{\circ} \mathrm{C}$. for a period of about 4 months in sealed ampoules (AprilJuly, 1954).

No tendency to hypersensitivity among the heated antigens was revealed in the testing of 120 routine negative sera. BFP sera were not tested, and the heated and non-heated antigens were not subjected to nephelometric evaluation.

(1) CF Antigens.-The effect of heating was tested in comparative CF experiments, using heated and non-heated samples.

Fig. 4 shows the results of the comparison between the reference antigen and the test antigen with normal lecithin concentration, heated and non-heated.

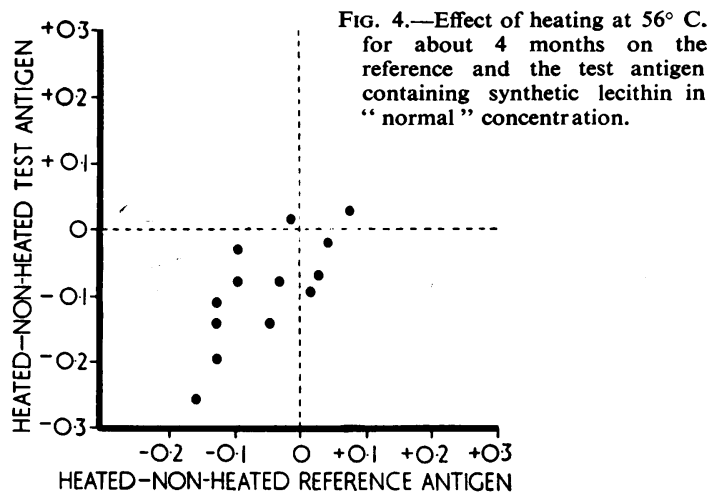

The differences between the titres obtained with heated and non-heated antigens are plotted against each other. Altogether, thirteen syphilitic sera were tested with the four antigens ; five of the sera were tested twice, and for these the average titres are indicated on the Figure, each point standing for one serum.

It is evident that both antigens become less sensitive after heating, the majority of the points being located in the lower left quadrant containing negative differences between heated and non-heated antigens. The average loss in sensitivity is greater with the test antigen : about $0 \cdot 1 \log _{10}$ value with a range of 0.3 for the test antigen and about 0.05 with the same range for the reference antigen. The test antigen with half concentration of lecithin was accidentally lost. The results with the test antigen with double concentration are shown in Fig. 5.

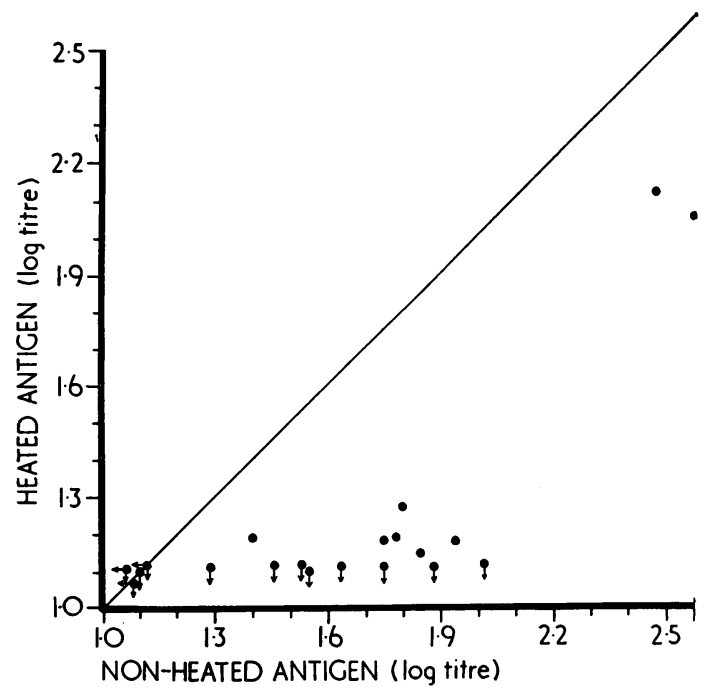

Fig. 5.-Effect of heating at $56^{\circ} \mathrm{C}$. for about 4 months of the test antigen containing synthetic lecithin in double concentration. 
Altogether, twenty syphilitic sera were tested with heated and non-heated antigens ; the corresponding titres are plotted against each other in Fig. 5, each point indicating the result with one serum. In this case a great loss in sensitivity is observed with the heated antigen ; in many cases the reactivity became so weak that the titre could only be evaluated as being below this limit. Thus, it was impossible to compute the average decrease, the individual decreases in titre varying from 0.2 to $\geqslant 0.9$.

(2) VDRL Antigens. - In previous experiments the VDRL test antigen was found less sensitive than the reference antigen (Reyn and Bentzon, in the press). In addition, the floccules were smaller with the test antigen than with the reference antigen. Heating the antigen suspensions to $56^{\circ} \mathrm{C}$. for periods up to 4 hours did not improve flocculation.

After storage at $56^{\circ} \mathrm{C}$. for 4 months, the VDRL test antigen showed a non-significant average decrease of 0.015 ; however, a significant decrease could be demonstrated in some of the sera. Similarly, with the reference antigen, a slight but weaker tendency to decreasing sensitivity after heating was observed, and in this case also the decrease was differently registered in the individual sera.

\section{Summary and Discussion}

Natural egg lecithin was replaced by crystalline synthetic $\mathrm{L}-\alpha$-dimyristoyl lecithin in complementfixation antigens with varying lecithin concentrations, the cardiolipin and cholesterol concentrations being constant ; thus, the cardiolipin/ lecithin ratio was varied as well as the absolute lecithin concentration.

The average sensitivity, as measured by the average titres of five freeze-dried syphilitic sera, was found to be slightly lower for the test antigen containing " normal" concentration of synthetic lecithin (molecule by molecule) than for the reference antigen.

Starting from the " normal" concentration, the average sensitivity decreased with increased concentration of synthetic lecithin, and it increased with decreasing lecithin concentration down to about $1 / 8$ of the "normal" concentration; 1/16 showed about the same level as $1 / 8$, but the antigen without any lecithin at all (Cardchol) showed an average sensitivity nearly as low as that of the antigen with double concentration.

The repeated use of the same freeze-dried sera made it possible to investigate how the variation in concentration influenced the range of deviation from the reference antigen in individual sera. This range was smallest with the test antigen with " normal" concentration of synthetic lecithin, and showed increasing values with both increasing and decreasing lecithin concentrations.

The antigen with a half concentration of synthetic lecithin had about the same level of sensitivity as the reference antigen, but at the same time the range of deviation of the individual sera was greater than that found with " normal" concentration. Thus, it was impossible to obtain at the same time the same average sensitivity and the slightest possible range of deviation of individual sera. This applies to the five syphilitic sera.

In addition two strongly biological false positive sera (BFP) were repeatedly tested with some of the antigens; half concentration of synthetic lecithin resulted in nearly complete lack of reaction, but the reactivity greatly increased with decreasing lecithin concentrations. The day-to-day variation of these sera was much greater than that of the specific sera.

The results obtained with the BFP sera indicate that BFP sera may be even more susceptible than syphilitic sera to changes both in the type of lecithin and in the lecithin concentration. Neither with the VDRL nor with the CF antigens containing synthetic lecithin was hypersensitivity found in routine negative sera.

The nephelometric maturation curve for the reference antigen was rather close to that of the test antigen with " normal" lecithin $w / v$ (weight/ volume) concentration, being located slightly lower than the curve for this antigen; this was in conformity with the serological findings.

However, the curve for half concentration was located much higher up, though the antigen showed about the same average sensitivity as that of the reference antigen. At concentrations of synthetic lecithin higher than $1 / 8$ of the "normal" concentration the nephelometric values and the serological findings were correlated in such a way that increasing lecithin concentration was accompanied by decreasing sensitivity. This correlation suggests the possibility of nephelometric control of cardiolipin antigens. This problem will be subjected to a closer analysis in a subsequent paper.

The range of deviation from the reference antigen for the individual sera was greatest in the case of the antigen containing double concentration of synthetic lecithin. Comparing the CF experiments performed in April and in October, this range was increased in October, indicating the possibility of an incipient deterioration of this antigen (Fig. 1).

Hence, the keeping qualities of antigens prepared with synthetic lecithin were further investigated in both VDRL and CF experiments. From previous investigations it was known that cardiolipin antigens prepared with natural lecithin withstand heating at 
$37^{\circ} \mathrm{C}$. for 3 months (WHO Report, 1953). Therefore, it was decided to expose both the reference and test antigens to a higher temperature for a longer time, i.e., $56^{\circ} \mathrm{C}$. for about 4 months. The VDRL antigens with " normal" concentration showed an insignificant loss in sensitivity, the reference antigen being less influenced than the test antigen; the latter showed a significant loss in some of the sera tested. The order of magnitude of the average loss in sensitivity was about $0.015 \log _{10}$ value in the case of the test antigen. All the CF antigens subjected to heating showed decreased sensitivity; those containing natural lecithin showed the smallest decrease. The average loss in sensitivity was about $0 \cdot 1 \log _{10}$ value for the test antigen with " normal " concentration and about $0.05 \log _{10}$ value for the reference antigen. The decrease of the test antigen with the double concentration ranged from 0.2 to $\geqslant 0.9$ in $\log _{10}$ value, thus being by far the greatest loss observed, and indicating the significance of the lecithin for the keeping quality of a cardiolipin antigen. Impurities may be the cause of the increasing loss in sensitivity with increasing lecithin concentration.

From our experiments we must conclude that when synthetic lecithin is substituted for natural egg lecithin it is impossible to obtain a complementfixation antigen of " exactly the same sensitivity for all sera". We can obtain the same average sensitivity, but at the same time the ratio between the titres for the individual sera will change. The greatest change observed was that for antigen with a double concentration of synthetic lecithin, the log difference of the ratio between the titres of serum 119 and 120 being $0.139 \log _{10}$ less in value than that found with the reference antigen. In other words, the inter serum ratio was changed by a factor of $1: 1 \cdot 4$.

Although it was shown that synthetic, crystalline, L- $\alpha$-dimyristoyl lecithin cannot replace natural lecithin in CF cardiolipin antigens without either a slight loss in sensitivity or an altered classification of sera in quantitative experiments, the results encourage further investigations with other batches of synthetic lecithin.

Assuming preliminary experiments to show the serological fitness of one batch of synthetic lecithin, additional important requirements in this substance would be constancy from one batch to the other, purity during storage, and relatively low cost.

The work with synthetic lecithins offers exicellent possibilities for the study of the combination of antigen and antibody, especially the significance of the nature of the fatty acids for this combination.

\section{REFERENCES}

Baer, E. (1952). J. biol. Chem.. 198, 853.

(1953). J. Amer. chem. Soc., 75, 621.

and Kates, M. (1949). Science, 109, 31.

-(1950). J. Amer. chem. Soc., 72, 942.

, and Martin, F. (1951). J. biol. Chem., 193, 835.

and Maurukas, J. (1952). J. Amer. chem. Soc., 74, 158.

Brown, R. (1946). J. immunol., 52, 17.

Faure, M. (1949). Ann. Inst. Pasteur, 76, 465.

(1956). Bull. Wld Hlth Org., 14. In the press.

- , and Maréchal, J. (1952). Ann. Inst. Pasteur, 82, 738.

Finney, D. J. (1947). “ Probit Analysis", p. 39. University Press, Cambridge.

Hartmann, J. (1955). Acta path. microbiol. scand., 36, 82.

- and Reyn, A. (1955). Ibid., 36, 129.

,-- , and Schmidt, H. (1953). Atti del VI Congresso internazionale di microbiologia, 2, 192. Riassunti delle Comunicazioni, vol. 1, p. 486 [No. 336]. Rome, 1953.

Kline, B. S. (1950). Amer. J. Syph., 34, 460.

-(1954a). Ibid., 38, 578.

- (1954b). Amer. J. clin Path., 24, 859.

- (1955). Ibid., 25, 971.

Lundbäck, H. (1952). "Studies on the Wassermann Reaction." Thesis. Uppsala.

Nelson, R. A., and Mayer, M. M. (1949). J. exp. Med., 89, 369.

Pangborn, M. C., Maltaner, F., Tompkins, V. N., Beecher, T., Thompson, W. R., and Flynn, M. R. (1951). "Cardiolipin Antigens." World Health Organization, Monograph Series, No. 6. Geneva.

Reyn, A., and Bentzon, M. W. (1956). Bull. Wld Hlth Org., 14. In the press.

Rosenberg, A. A. (1949). J. Vener. Dis. Inform., 30, 194.

Schmidt, H. (1951). British Journal of Venereal Diseases, 27, 23.

-(1955a). Acta path. microbiol. scand., 36, 141.

(1955b). Ibid., 36, 147.

, and Lundbäck, H. (1954). Ibid., 34, 509.

,-- , and Reyn, A. (1953). Atti del VI congresso internazionale di microbiologia, 2, 194. Riassunti delle Comunicazioni, vol. 1, p. 562 [No. 386]. Rome, 1953.

Tonks, D. B., and Allen, R. H. (1953). Science, 118, 55.

- - (1955). British Journal of Venereal Diseases, 31, 180. - , and Fowler, E. (1955). Symposium on recent advances in the study of veneral disease, 1955.

WHO/VD/SERO/65. (1953). Report on Storage Experiments with Cardiolipin, Lecithins and Cardiolipin Antigens. 